\title{
Decoherence at zero temperature
}

\author{
G W Ford ${ }^{1}$ and R F O'Connell ${ }^{2}$ \\ School of Theoretical Physics, Dublin Institute for Advanced Studies, 10 Burlington Road, \\ Dublin 4, Republic of Ireland
}

Received 28 February 2003, accepted for publication 14 March 2003

Published 16 October 2003

Online at stacks.iop.org/JOptB/5/S609

\begin{abstract}
Most discussions of decoherence in the literature consider the high temperature regime but it is also known that, in the presence of dissipation, decoherence can occur even at zero temperature. Whereas most previous investigations all assumed initial decoupling of the quantum system and bath, we consider that the system and environment are entangled at all times. Here, we discuss decoherence for a free particle in an initial Schrödinger cat state. Memory effects are incorporated by use of the single relaxation time model.
\end{abstract}

Keywords: Decoherence, dissipation, zero-temperature heat bath

\begin{abstract}
Most discussions of decoherence obtain results for decoherence decay times which are proportional to the inverse of $T$ or $T^{1 / 2}$, where $T$ is the temperature, and which apply only to the high temperature regime, $k T \gg \hbar \gamma$, where $\gamma$ is a typical dissipative decay rate [1-5]. In fact, whereas most papers in the early literature give decay rates proportional to $\gamma^{-1}$, we showed that decoherence can occur at high temperature even for vanishingly small dissipation $[4,5]$. For a pedagogical discussion of the high $T$ regime, we refer the reader to [6]. Here, we look at the opposite end of the temperature spectrum and present explicit results at temperature zero. In contrast to the case for the high temperature regime, we find that a dissipative environment is necessary to achieve decoherence.
\end{abstract}

The zero temperature case was also considered by others, most notably by Walls and Milburn [2]. They considered a 'Schrödinger cat' state consisting of a pair of coherent states of an oscillator. They solved the master equation to obtain an expression for the coherence attenuation coefficient, defined in terms of the off-diagonal elements of the density matrix. However, this approach is subject to serious assumptions: (a) initial decoupling of the system and environment; (b) weak coupling, which implies a restriction to a finite oscillator potential.

The assumption (a) used by Walls and Milburn and indeed many other investigators leads to results which are seriously at variance with a model in which the quantum particle and the heat bath are entangled at all times. In fact, at $T=0$, we are simply dealing with the zero point oscillations of

\footnotetext{
1 Permanent address: Department of Physics, University of Michigan, Ann Arbor, MI 48109-1120, USA.

2 Permanent address: Department of Physics and Astronomy, Louisiana State University, Baton Rouge, LA 70803-4001, USA.
}

the heat bath which are necessarily always entangled with whatever quantum particle is of interest. In fact, the results that we obtain here are applicable to the whole low temperature regime, defined by the inequality $k T \ll \hbar \gamma$. General results for all regimes, corresponding to arbitrary $T$ and $\gamma$, were obtained in our initial paper on this subject [4], where our starting point was the density matrix for the system of the particle coupled to the bath at all times. However, there explicit results were presented only for the Ohmic model in the high temperature regime $(k T \gg \hbar \gamma)$. Later, we showed that the same results could be obtained simply by starting with the wavefunction describing a pure Schrödinger cat state moving with an initial velocity $v$ [5]. This was then used to calculate the wavefunction and then the coordinate probability distribution at time $t$. Finally, this probability distribution was then averaged over a thermal distribution of initial velocities to obtain the probability distribution corresponding to a finite high temperature $T$. This led immediately to a quantitative measure of decoherence (the destruction of the interference pattern). In other words, the calculation of the decoherence decay time at high temperature could be carried out simply within the framework of elementary quantum mechanics and equilibrium statistical mechanics, with a result independent of $\gamma[5,6]$.

In contrast to the high temperature case, results at low temperature depend essentially on $\gamma$ and must be obtained within the framework of nonequilibrium statistical mechanics. Thus, we will now give a synopsis of our general model describing a system and its environment as being entangled at all times [4].

First, we emphasize that, not only are our results in general applicable to arbitrary dissipation and arbitrary 
temperature, but also they are not subject to the weak coupling approximation and so apply in the case of a free particle. Arbitrary dissipation refers to reservoir models which give rise to memory terms in the quantum Langevin equation (see (6) below and the subsequent discussion) which describes the quantum system in a dissipative environment. By contrast, the Ohmic model (the model considered by most authors) is the simplest dissipative model and corresponds to the lack of memory terms in the Langevin equation. As we shall see below, the Ohmic model has the difficulty that the mean square velocity at zero temperature is divergent and a cut-off must be supplied to make it finite. For this purpose we use the single relaxation time model (which reduces to the Ohmic model in the limit of zero relaxation time).

As in our previous discussions [4-6], we consider decoherence in terms of the simple problem of a free particle moving in one dimension that is placed in an initial superposition state ('Schrödinger cat' state) corresponding to a pair of Gaussian wavepackets, each with variance $\sigma^{2}$ and separated by a distance $d \gg \sigma$. For such a state the probability distribution at time $t$ can be shown to be of the form

$$
\begin{aligned}
& P(x, t)=\frac{1}{2\left(1+\mathrm{e}^{-d^{2} / 8 \sigma^{2}}\right)}\left\{P_{0}\left(x-\frac{d}{2}, t\right)+P_{0}\left(x+\frac{d}{2}, t\right)\right. \\
& \left.+2 \mathrm{e}^{-d^{2} / 8 w^{2}(t)} a(t) P_{0}(x, t) \cos \frac{[x(0), x(t)] x d}{4 \mathrm{i} \sigma^{2} w^{2}(t)}\right\},
\end{aligned}
$$

where $P_{0}$ is the probability distribution for a single wavepacket, given by

$$
P_{0}(x, t)=\frac{1}{\sqrt{2 \pi w^{2}(t)}} \exp \left\{-\frac{x^{2}}{2 w^{2}(t)}\right\} .
$$

Here and in (1) $w^{2}(t)$ is the variance of a single wavepacket, which in general is given by

$$
w^{2}(t)=\sigma^{2}-\frac{[x(0), x(t)]^{2}}{4 \sigma^{2}}+s(t)
$$

where $\sigma^{2}$ is the initial variance, $[x(0), x(t)]$ is the commutator, and

$$
s(t)=\left\langle\{x(t)-x(0)\}^{2}\right\rangle,
$$

is the mean square displacement. In (1) the first two terms within the braces correspond to the two wavepackets, centred at $\pm d / 2$, expanding independently, while the third term is the interference term. Decoherence refers to the destruction of interference, a measure of which is given by the attenuation coefficient $a(t)$ which can be defined as the ratio of the factor multiplying the cosine in the interference term to twice the geometric mean of the first two terms $[4,5]$. Using a method of successive measurements introduced by Ford and Lewis [7], in which a particle in equilibrium and entangled with the environment is placed in the initial state by a first measurement and then, after a time interval $t$, is probed by a second measurement, we obtained the following exact general formula for the attenuation coefficient $[4,7]$ :

$$
a(t)=\exp \left\{-\frac{s(t) d^{2}}{8 \sigma^{2} w^{2}(t)}\right\} .
$$

The quantities appearing in (3) and (4) are evaluated by use of the quantum Langevin equation [8], which is a Heisenberg equation of motion for $x(t)$, the dynamical variable corresponding to the coordinate of a Brownian particle interacting with a linear passive heat bath. For the case of a free particle, this equation for the stationary process has the well known form

$$
m \ddot{x}+\int_{-\infty}^{t} \mathrm{~d} t^{\prime} \mu\left(t-t^{\prime}\right) \dot{x}\left(t^{\prime}\right)=F(t),
$$

where $\mu(t)$ is the memory function and $F(t)$ is a fluctuating operator force with mean zero. The solution of the quantum Langevin equation (6) can be written as

$$
x(t)=\int_{-\infty}^{t} \mathrm{~d} t^{\prime} G\left(t-t^{\prime}\right) F\left(t^{\prime}\right),
$$

where $G(t)$, the Green function, can in turn be written as

$$
G(t)=\frac{1}{2 \pi} \int_{-\infty}^{\infty} \mathrm{d} \omega \alpha\left(\omega+\mathrm{i} 0^{+}\right) \mathrm{e}^{-\mathrm{i} \omega t},
$$

in which $\alpha(z)$ (the Fourier transform of the Green function) is the response function. For the free particle the response function has the general form

$$
\alpha(z)=\frac{1}{-m z^{2}-\mathrm{i} z \tilde{\mu}(z)},
$$

in which $\tilde{\mu}(z)$ is the Fourier transform of the memory function,

$$
\tilde{\mu}(z)=\int_{0}^{\infty} \mathrm{d} t \mu(t) \mathrm{e}^{\mathrm{i} z t}, \quad \operatorname{Im}\{z\}>0 .
$$

Using these results, we find that [8,9] the mean square displacement is given by the formula

$$
s(t)=\frac{2 \hbar}{\pi} \int_{0}^{\infty} \mathrm{d} \omega \operatorname{Im}\left\{\alpha\left(\omega+\mathrm{i} 0^{+}\right)\right\} \operatorname{coth} \frac{\hbar \omega}{2 k T}(1-\cos \omega t),
$$

while the commutator, which is temperature independent, is given by the formula

$$
\left[x\left(t_{1}\right), x\left(t_{1}+t\right)\right]=\frac{2 \mathrm{i} \hbar}{\pi} \int_{0}^{\infty} \mathrm{d} \omega \operatorname{Im}\left\{\alpha\left(\omega+\mathrm{i} 0^{+}\right)\right\} \sin \omega t .
$$

These expressions are valid for arbitrary temperature and arbitrary dissipation. (Indeed, with the appropriate expression for the response function, they are valid in the presence of an external oscillator potential.) In our earlier discussions, for brevity, we confined our attention to the case of high temperature. Here we consider the case of zero temperature. Most discussions in the literature are confined to the socalled Ohmic case, where $\tilde{\mu}(z)=\zeta$, the Newtonian friction constant but we analyse the more general case of the single relaxation time model [10]. This model corresponds to a memory function of the form

$$
\mu(t)=\frac{\zeta}{\tau} \mathrm{e}^{-t / \tau} \theta(t),
$$

where $\tau$ is the relaxation time of the bath and where $\theta$ is the Heaviside function. Note that in the limit $\tau \rightarrow 0$ this becomes the Ohmic memory function $\mu(t)=2 \zeta \delta(t) \theta(t)$. With this form of the memory function,

$$
\tilde{\mu}(z)=\frac{\zeta}{1-\mathrm{i} z \tau} .
$$


Next, with this form in the expression (9) for the response function, we find with $T=0$ that the expression (11) for the response can be expressed in the form

$$
s(t)=\frac{2 \hbar}{\pi \zeta} \frac{\Omega^{2} V(\gamma t)-\gamma^{2} V(\Omega t)}{\Omega^{2}-\gamma^{2}},
$$

while the expression (12) for the commutator becomes

$$
[x(0), x(t)]=\frac{\mathrm{i} \hbar}{\zeta} \frac{\Omega^{2}\left(1-\mathrm{e}^{-\gamma t}\right)-\gamma^{2}\left(1-\mathrm{e}^{-\Omega t}\right)}{\Omega^{2}-\gamma^{2}},
$$

where we have introduced the quantities

$$
\Omega=\frac{1+\sqrt{1-4 \zeta \tau / m}}{2 \tau}, \quad \gamma=\frac{1-\sqrt{1-4 \zeta \tau / m}}{2 \tau},
$$

and where [11]

$$
\begin{aligned}
& V(x)=\int_{0}^{\infty} \mathrm{d} y \frac{x^{2}}{y\left(y^{2}+x^{2}\right)}(1-\cos y) \\
& =\log x+\gamma_{\mathrm{E}}-\frac{1}{2}\left[\mathrm{e}^{-x} \operatorname{Ei}(x)+\mathrm{e}^{x} \operatorname{Ei}(-x)\right] \\
& =-\left(\log x+\gamma_{\mathrm{E}}\right)(\cosh x-1) \\
& \quad-\frac{1}{2}\left[\mathrm{e}^{-x} \sum_{n=1}^{\infty} \frac{x^{n}}{n ! n}+\mathrm{e}^{x} \sum_{n=1}^{\infty} \frac{(-x)^{n}}{n ! n}\right] .
\end{aligned}
$$

Here $\gamma_{\mathrm{E}}=0.577215665$ is Euler's constant. Note that the sums in (18) are absolutely convergent. We should point out that the quantities $\Omega$ and $\gamma$ were referred to as $\gamma_{+}$and $\gamma_{-}$in [10]. We feel that the present choice is more suggestive since, for $\tau \rightarrow 0$, we have $\Omega \rightarrow 1 / \tau$ and $\gamma \rightarrow \zeta / m$.

Note the expansions [12], for small $x$,

$$
V(x) \cong-\frac{1}{2} x^{2}\left(\log x+\gamma_{\mathrm{E}}-\frac{3}{2}\right)
$$

Also, asymptotically, for large $x$,

$$
V(x) \sim \log x+\gamma_{\mathrm{E}}-\frac{1}{x^{2}}-\frac{3 !}{x^{4}}-\frac{5 !}{x^{6}} .
$$

For very short times $(t \ll \tau)$, one can show in general that

$$
s(t) \cong\left\langle v^{2}\right\rangle t^{2}
$$

where $\left\langle v^{2}\right\rangle$ is the mean square velocity, given for the single relaxation time model by

$$
\left\langle v^{2}\right\rangle=\frac{\hbar \gamma \Omega}{\pi m(\Omega-\gamma)} \log \frac{\Omega}{\gamma} \cong-\frac{\hbar \zeta}{\pi m^{2}} \log \frac{\zeta \tau}{m} .
$$

We note that, for $\tau \rightarrow 0$ (corresponding to the Ohmic model), the mean square velocity, given in (22), is logarithmically divergent. The conclusion is that, in order to obtain finite results for $\left\langle v^{2}\right\rangle$ at short time, one must use a cut-off model, such as the single relaxation time model used here.

For intermediate times $\left(\tau \ll t \ll(\zeta / m)^{-1}\right)$, we obtain

$$
s(t) \cong-\frac{\hbar \zeta}{\pi m^{2}} t^{2}\left\{\log \frac{\zeta t}{m}+\gamma_{\mathrm{E}}-\frac{3}{2}\right\},
$$

which is the Ohmic model result. We can compare these results with the weak coupling result which for a free particle leads to $s(t)$ being identically zero. (For a free particle the mean square velocity vanishes at zero temperature.)
Thus, to investigate the decay of coherence (which is generally a short time phenomenon) in both time ranges, we consider the behaviour for times $\zeta t / m \ll 1$. In this case, the commutator becomes that of a non-interacting particle:

$$
[x(0), x(t)] \cong \mathrm{i} \frac{\hbar t}{m} .
$$

Hence, it is clear that for very short times the wavepacket width is the initial width:

$$
w^{2}(t) \cong \sigma^{2} .
$$

Thus, the attenuation coefficient (5) for short times takes the form

$$
a(t) \cong \exp \left\{-\frac{s(t) d^{2}}{8 \sigma^{4}}\right\},
$$

where $s(t)$ is given by (21) and (23). Hence, in particular, we can write

$$
a(t)=\exp \left\{\left(\frac{t}{\tau_{0}}\right)^{2} \log \frac{\zeta \tau}{m}\right\}, \quad t \ll \tau,
$$

and

$$
\begin{aligned}
a(t) & =\exp \left\{\left(\frac{t}{\tau_{0}}\right)^{2}\left[\log \frac{\zeta t}{m}+\gamma_{\mathrm{E}}-\frac{3}{2}\right]\right\}, \\
\tau & \ll t \ll(\zeta / m)^{-1},
\end{aligned}
$$

is the Ohmic result, with

$$
\tau_{0} \equiv \frac{m \sigma^{2}}{d} \sqrt{\frac{8 \pi}{\hbar \zeta}}
$$

The characteristic time for decoherence to occur, $\tau_{\mathrm{d}}$, is defined, as usual [4-6], as the time at which $a(t)=\exp (-1)$. Hence, for example it follows from (27) that, for $t \ll \tau$,

$$
\tau_{\mathrm{d}}=\tau_{0}|\log (\zeta \tau / m)|^{-1 / 2}<\tau_{0}
$$

where the inequality sign follows from the fact that $\zeta t / m \ll 1$. It is also clear that $\tau_{\mathrm{d}}<\tau_{0}$ in the case $t \gg \tau$.

Next, we consider some numerical results. As mentioned above, here $\zeta / m \approx \gamma$ and, as a point of reference, it is useful to note that

$$
\frac{k T}{\hbar \gamma}=\frac{T(K)}{\gamma\left(10^{11} \mathrm{~s}^{-1}\right)} \text {. }
$$

Now, one of the few experimental investigations of decoherence is the work of Myatt et al [13] on the decoherence of trapped ${ }^{9} \mathrm{Be}^{+}$ions for which $\gamma$ values of $\approx 6 \times 10^{3}$ are quoted. It is clear from (31) that for such low values of $\gamma$ we are in the low temperature regime even for $T$ values which would normally be considered very high. Finally, as an illustration, if we consider a ${ }^{9} \mathrm{Be}$ ion with $\gamma \approx 6 \times 10^{3}$ and if we take $\sigma=1 \AA$ and $d=1 \mathrm{~cm}$ then, from (29), we obtain a $\tau_{0}$ value of $\approx 6 \times 10^{-16} \mathrm{~s}^{-1}$ and, as mentioned above, $\tau_{\mathrm{d}}$ will be even smaller; i.e. decoherence occurs in a very short time even at zero temperature and indeed throughout the whole low temperature regime.

\section{Acknowledgment}

The authors wish to thank the School of Theoretical Physics, Dublin Institute for Advanced Studies, for their hospitality and the Director, Dr Tony Douglas, for an enlightening remark concerning the evaluation of the integral in equation (18). 


\section{References}

[1] Giulini D, Joos E, Kiefer C, Kupisch J, Stamatescu I-O and Zeh H D 1996 Decoherence and the Appearance of a Classical World in Quantum Theory (New York: Springer)

[2] Walls D F and Milburn G J 1985 Phys. Rev. A 312403

[3] Zurek W H 1991 Phys. Today 4436

[4] Ford G W, Lewis J T and O'Connell R F 2001 Phys. Rev. A 64 032101

[5] Ford G W and O'Connell R F 2001 Phys. Lett. A 28687

[6] Ford G W and O'Connell R F 2002 Am. J. Phys. 70319

[7] Ford G W and Lewis J T 1986 Probability, Statistical Mechanics, and Number Theory (Advances in Mathematics Supplemental Studies vol 9) (Orlando, FL: Academic)
[8] Ford G W, Lewis J T and O'Connell R F 1988 Phys. Rev. A 37 4419

[9] Ford G W and O'Connell R F 1989 J. Stat. Phys. 57803

[10] Ford G W and O'Connell R F 2001 Phys. Rev. D 64 105020 especially the appendix

[11] Bateman Manuscript Project 1954 Tables of Integral Transforms vol 1, ed A Erdélyi (New York: McGraw-Hill)

[12] Bateman Manuscript Project 1954 Higher Transcendental Functions vol 2, ed A Erdélyi (New York: McGraw-Hill)

[13] Myatt C J, King B E, Turchette Q A, Sackett C A, Kielpinski D, Itano W M, Monroe C and Wineland D J 2000 Nature $\mathbf{4 0 3} 269$ 